Sind die Piraten also eine Partei der internetaffinen Postmaterialisten? So plausibel diese These klingt, die erklären könnte, weshalb die Piratenpartei über ihr eigentliches Milieu hinaus mobilisieren kann: Die Daten aus den Wählerbefragungen sprechen zumindest teilweise eine andere Sprache. Denn wie geht die Hypothese, dass die Piratenpartei in erster Linie die internetaffinen Postmaterialisten mobilisiere, mit dem Umstand zusammen, dass dieselbe Partei bei den Wahlen nach Berlin einen überdurchschnittlich hohen Anteil an Arbeitslosen unter ihren Wählern aufweist? Hier braucht es doch einiges an Phantasie, um den Erfolg der Piratenpartei nicht (auch) in Verbindung mit dem Protestmotiv zu bringen. Entsprechend abenteuerlich ist Appelius' und Fuhrers Behauptung, dass „die Kategorie der Arbeitslosen bei der Piraten-Anhängerschaft nicht in erster Linie aus den klassischen HartzIV-Empfängern rekrutiert, sondern eher aus einer Gruppe, die gut ausgebildet ist, aber (noch) keine gesicherten Arbeitsverhältnisse hat" (S. 64).

Bleibt noch die Frage zum Potential von Liquid Democracy: Kann dieses Konzept Umsetzungsprobleme der direkten oder sogar deliberativen Demokratie lösen? Appelius und Fuhrer sind optimistisch. Sie gehen zurück auf die Urdemokratie Athens und versuchen zu zeigen, dass eine ähnliche Vorstellung von Demokratie, wie sie der Idee der „flüssigen Demokratie“ innewohnt, schon einmal erfolgreich umgesetzt wurde. Sie nennen jedoch zwei Probleme der direkten Demokratie Athens: Erstens, dass diese, wie auch Rousseau zu bedenken gab, nur in einer relativ kleinen Gemeinschaft umsetzbar sei. Dafür böten „Tools“ im Geiste von Liquid Democracy wie LiquidFeedback eine Lösung, indem Kommunikations- und Abstimmungsprozesse in einen virtuellen Raum verlagert werden. Zweitens drohte der direkten Demokratie athenischen Typs die Machtkonzentration bei Demagogen. Hierfür, so geben Appelius und Fuhrer zu, hätte auch das Konzept der Liquid Democracy noch keine Lösung bereit.

Insgesamt ist es der Politikwissenschaft mit Bezug auf die Piratenpartei gelungen, sehr schnell einige interessante Antworten auf ein neues Phänomen zu geben. Weiterführende Forschung in diese Richtung ist jedoch erwünscht. Insbesondere fehlt es nach wie vor an einer fundierten wahlsoziologischen Analyse, die eine überzeugende Antwort auf die Dynamik des Wahlerfolgs der Piratenpartei gibt. Auch was den demokratietheoretischen Wert des Liquid Democracy-Konzepts betrifft, bleiben noch viele Fragen offen. So wäre es interessant, dieses nicht nur im Lichte von Idealtypen partizipativer Demokratie zu betrachten, sondern mit Bezug auf die real existierenden (semi-)direkten Demokratien zu reflektieren.

Oliver Strijbis

\title{
Piratenpartei: weitere Erschließung eines politischen Phänomens
}

Bieber, Christoph und Claus Leggewie (Hrsg.): Unter Piraten. Erkundungen in einer neuen politischen Arena, transcript, Bielefeld 2012, 248 Seiten, € 19,80.

Niedermayer, Oskar (Hrsg.): Die Piratenpartei, VS Verlag für Sozialwissenschaften, Wiesbaden 2012, 200 Seiten, $€ 24,95$.

Berlin am 18. September 2011, 18 Uhr: Die erste Hochrechnung zur Berliner Abgeordnetenhauswahl geht von 8,9 Prozent der Zweitstimmen für die Piratenpartei aus. Dies ist das singuläre Ereignis, das eine intensive wissenschaftliche Beschäftigung mit dem jungen Phä- 
nomen unabdingbar gemacht hat. In den ersten fünf Jahren nach der Gründung der Piratenpartei Deutschland war die Forschungsliteratur zum Thema überschaubar. Mit dem genannten Wahlerfolg setzte eine Publikationsflut ein. Ganzheitlich und analytisch sind die wenigsten dieser Veröffentlichungen. ${ }^{1}$ Im Sommer 2012 sind nun gleich zwei Sammelbände erschienen, die diese Eigenschaften im besten Sinne aufweisen.

Der erste ist von Christoph Bieber und Claus Leggewie herausgegeben worden. Der Titel „Unter Piraten. Erkundungen in einer neuen politischen Arena" verdeutlicht bereits den Anspruch. Die übergreifende Zielsetzung des Bandes beschreibt Christoph Bieber als das Bemühen, am Ende der „Inkubationsphase ,digitaler Demokratie“ die Innovationen im Bereich der politischen Kultur zu analysieren (S. 17 f.). Zwischen einer „ermunternde[n] Einleitung“ (Christoph Bieber) und einer „skeptische[n] Nachbetrachtung“ (Claus Leggewie) steuern weitere 21 Autoren insgesamt 18 Beiträge zu dem Sammelband bei. Die Gruppe wird von Politikwissenschaftlern und Soziologen dominiert, schließt aber auch Journalisten, studierte Philosophen, Rechts- und Wirtschaftswissenschaftler sowie Wissenschaftler mit dem Schwerpunkt Kommunikation ein. Diese interdisziplinäre Ausrichtung bringt eine heterogene Bandbreite an Themen hervor, die unter drei Oberbegriffen subsumiert werden: Entern, Ändern und Neustart.

Der erste Block beleuchtet die „Bewegungsimpulse“, die von der Piratenpartei ausgehen und das „Motiv“ ihres „Eindringens in die politische Sphäre“. „Betrachtet man die Piraten als Bewegungs- und Milieupartei, wird viel klarer, worin die für eine Parteineugründung zweifelsohne erheblichen Ressourcen und Energien wurzeln konnten" (Alexander Hensel, S. 45). Erweitert wird dieser Block durch einen Blick in die Wahrnehmung und das Selbstverständnis der Piraten. Dort ist zum einen der „Syllogismus [...]: Piraten kennen sich mit Computern aus. Wer sich mit Computern auskennt, ist ein Nerd. Also sind Piraten Nerds“ (Mathias Mertens, S. 56) und zum anderen eine Betrachtung zu Orange als Lagerfarbe „danach ergäben die Summanden Rot (sozial) und Gelb (liberal) die Wunschsumme Orange“ (Claudio Gallio, S. 84) - vorzufinden.

Im zweiten Abschnitt werden die „Programmatischen Konturen“ und das „Koordinatensystem“ des „Wertehorizonts“ der Partei nachvollzogen. Gleich im ersten Beitrag wird vielleicht der Kernpunkt des Programms herausgearbeitet: Plattformneutralität. Was sich zunächst nicht politisch anhört, lässt sich leicht in barrierefreien Zugang zur Gesellschaft und zu Information aller Art übersetzen. Konzepte wie das bedingungslose Grundeinkommen, kostenloser Nahverkehr, Freiheit im Netz etc. fallen unter diesen Programmpunkt (Michael Seemann, S. 92 - 95). In diesem Abschnitt findet sich dann ebenfalls eines der am meisten medial aufgearbeiteten Themen der Partei: das Fehlen von Frauen. Gerade in diesem Bereich gilt es laut den Autoren die hohe Heterogenität der Parteimitglieder zu beachten und nicht zuletzt den „Bewegungscharakter“ zu akzeptieren (Jasmin Siri und Paula-Irene Villa, S. 156).

Abschließend stellt sich den Autoren die Frage nach den „Schnittstellen“ und „Anschlussflächen “ der Änderungsimpulse, die von der Partei auf die Parteienlandschaft und das politische System ausgehen. Die zunehmende Beschleunigung des Alltags und die bisher fehlende Anpassung der parlamentarischen Demokratie liefern eine Begründung für den Erfolg der Partei (Beiträge von Jörn Lamla und Hartmut Rosa sowie Karl-Rudolf Korte). Die

1 Eine zu nennende Ausnahme ist: Recep Gürbüz, Piratenpartei: Genese - Ziele - Struktur - Zukunftsaussichten, Wiesbaden 2011. 
wichtige Frage, ob sich dieser Erfolg konservieren lässt, kann selbstverständlich nicht abschließend entschieden werden. Jedoch, so einer der Autoren, gelingt dies am ehesten, wenn sie dem Wähler weiterhin als thematisch und organisatorische Alternative zu den etablierten Parteien erscheinen kann (Stefan Marschall, S. 222).

Festzuhalten ist, dass eine politikwissenschaftliche Betrachtung vor allem im dritten Abschnitt stattfindet. Doch die Leistung des Bandes ist gerade, dass er zahlreiche Elemente, die das Phänomen ausmachen, losgelöst vom Ganzen zusätzlich interdisziplinär betrachtet und damit die Kernbegriffe, mit denen die Partei operiert, die von den Medien zum Teil verkürzt oder abgewandelt verwendet werden, klar zeichnet.

Der zweite Sammelband wurde von Oskar Niedermayer herausgegeben. Es handelt sich um eine Fortführung seiner Forschung zur Piratenpartei, über die er bereits Ende 2010 einen Artikel in der ZParl veröffentlicht hatte. ${ }^{2}$

Ein erster Blick ins Inhaltsverzeichnis unterstreicht das Engagement des Herausgebers in diesem Themenfeld. So sind fünf der 13 Beiträge aus seiner Feder. Darüber hinaus finden sich zahlreiche Autoren, die schon zur Piratenpartei publiziert haben. Die zentrale Zielsetzung des Bandes ist eine umfassende Analyse der Partei und der Rahmenbedingungen, die zu ihrem Erfolg geführt haben. Dessen Messung wird durch die Annahme möglich, dass Parteien in ihrem „Lebenszyklus“ Karrierestufen überspringen oder dies eben nicht tun (Oskar Niedermayer, S. 9 - 13).

In der Gliederung wird zunächst die Vorgeschichte, in Form der Urheberrechtsdebatte in Schweden und dem Auf und Ab der dortigen Piratenpartei, abgebildet (Henning Bartels). Dem schließt sich ein zeitlicher Abriss der Entwicklung in Deutschland an. Hierbei wird gleich einem Hürdenlauf der Weg von der ersten Wahlteilnahme im Jahr 2008 (1. Karrierestufe), über die Wettbewerbsbeeinflussung nach der Europawahl 2009 (2. Karrierestufe), zur parlamentarischen Repräsentation im Abgeordnetenhaus in Berlin 2011 (3. Karrierestufe) und der bisherige Höhepunkt, die koalitionsstrategische Inklusion nach dem Scheitern der rot-grünen Sondierungsgespräche in Berlin 2011 (4. Karrierestufe), dargestellt (Oskar Niedermayer, S. 33 - 49). Neben der zutreffenden Beobachtung, dass Piratenwähler ,jung, männlich und netzaffin" sind, kommt die Analyse zu dem Schluss, dass die Partei vor allem Randwähler anspricht (Oskar Niedermayer, S. 73).

Die politische Verortung, die sich als noch unentschieden, aber eher links subsumieren lässt, und die Organisationsstruktur der Partei runden den primär deskriptiven Teil des Bandes ab (Beiträge von Stefanie Haas, Richard Hilmer, Oskar Niedermayer).

Die personellen Überschneidungen zwischen den Autoren der beiden Bände wird am Beitrag zum Kommunikationsmanagement der Partei deutlich; dieser wurde von Christoph Bieber und Markus Lewitzki verfasst. Die Autoren erweitern in ihrem Fazit die zuvor getroffenen Aussagen zum Charakter der Parteiorganisation. Die Piratenpartei hat kein völlig neues Organisationsmodell entwickelt, sondern den Wandel der „Parteien in der Mediendemokratie", wie ihn Ulrich von Alemann und Stefan Marschall schon 2002 beschrieben haben, fortgeführt, „allerdings unter den Bedingungen digitaler, interaktiver Medien und vernetzter Öffentlichkeit“ (Christoph Bieber und Markus Lewitzki, S. 122).

Einer besonders interessanten Frage stellt sich der Beitrag von Marc Debus und Thorsten Faas. Etwas vereinfacht: Wo ist die Lücke, in die die Piratenpartei programmatisch hinein-

2 Vgl Oskar Niedermayer, Erfolgsbedingungen neuer Parteien im Parteiensystem am Beispiel der Piratenpartei Deutschland, in: ZParl, 41. Jg. (2010), H. 4, S. 838 - 854. 
stößt, und welche Koalitionsoptionen ergeben sich hieraus? Die Autoren zeigen, dass „die Piraten in innen-, rechts- und gesellschaftspolitische Fragen explizit progressiv-libertär ausgerichtet sind, es in Fragen der Wirtschafts- und Sozialpolitik jedoch Anzeichen dafür gibt, dass die Piraten eher moderate Position einnehmen“ (S. 208).

Die Genderdebatte, die Plattformneutralität - also die Programmatik der Partei -, die Arbeit der Berliner Landtagsabgeordneten der Piraten und die Reaktion der anderen Parteien in der "Netzpolitik“ runden die Analyse ab (Beiträge von Manuela S. Kulick, Felix Neumann, Carsten Koschmiede und Oskar Niedermayer).

In einem vergleichenden Fazit ist zunächst festzuhalten, dass beide Publikationen die Landtagswahlen in Schleswig-Holstein und Nordrhein-Westfalen nicht betrachten. Oskar Niedermayer bemerkt hierzu zu Recht, dass „,bei einem normalen Verlauf der jeweiligen Legislaturperioden in den Bundesländern [...] im Jahre 2012 keine einzige Landtagswahl“ (Oskar Niedermayer, S. 49) stattgefunden hätte. Ausgehend von den jeweiligen Vorarbeiten war es daher folgerichtig, Berlin als Höhepunkt und wichtigsten Datenpunkt zu nutzen, ohne die weitere Entwicklung - der Wahltermin für Schleswig-Holstein war bereits seit dem 7. Juni 2011 bekannt - in den Mittelpunkt zu rücken. Die große Leistung beider Bücher ist hiervon unabhängig, dass Berlin nicht als Anfangspunkt eines „Hypes“ betrachtet wird. Vielmehr ist, vor allem bei Bieber/Leggewie, ein breiter Erklärungsansatz gewählt worden, der das Phänomen mit seinen gesellschaftlichen Vorläufern beziehungsweise seiner parteipolitischen Geschichte abbildet. Die einzelnen Artikel in dem von Niedermayer herausgegebenen Band stehen stärker unter politikwissenschaftlichen Fragestellungen, als dies bei dem interdisziplinären Ansatz von Bieber/Leggewie der Fall ist. Damit wirkt der erste stärker als Ist-Stands-Beschreibung, während der zweite alternative Betrachtungsweisen und perspektivische Forschungsfragen skizziert. Als Grundlagenliteratur zur Piratenpartei sind beide uneingeschränkt zu empfehlen.

Christian Nestler

\section{Piratenpartei: Politische Kommunikation 2.0?}

Odenbach, Jan: Partei, Netz, Netzpartei. Meinungs- und Willensbildung in der Piratenpartei, Springer VS, Wiesbaden 2012, 120 Seiten, € 29,95.

Wenzlaff, Oliver: Piratenkommunikation. Was die Eliten in Politik und Wirtschaft von den Piraten lernen können, ambition Verlag, Berlin 2012, 224 Seiten, € 24,99.

Kommunikation egal in welcher Form ist der wichtigste Bestandteil jedes menschlichen Zusammenlebens. Politische Kommunikation nimmt in dieser Hinsicht eine Sonderrolle ein, denn sie liegt ständig unter einem medialen Vergrößerungsglas. Im Jahr 2012 sind zwei Bücher erschienen, die die „neuartige“ Kommunikation der Piratenpartei nach Innen und Außen betrachten.

Das erste wurde von Jan Odenbach verfasst und wirft bereits im Titel die zentrale Frage und möglicherweise deren Antwort auf. „Partei, Netz, Netzpartei“ lässt sich zunächst in die Gleichung Partei + Netz = Netzpartei überführen (S. 17). Die als Band 31 in der Reihe "College“ des Springer VS Verlags erschienene Publikation basiert auf der Diplomarbeit des 